\title{
DISTORSIONES QUE ALTERAN LA MORFOLOGÍA DEL ELECTROCARDIOGRAMA, CAUSADAS POR DESPLAZAMIENTO DE LÍNEA DE BASE Y LA RED ELÉCTRICA
}

1 proceso de adquisición del electrocardiograma está sujeto a la captura de
diferentes tipos de señales que se adicionan causando distorsiones. Este tipo de señales son tratadas con filtros analógicos que incrementan el tamaño de los diseños electrónicos y el consumo de energía. A pesar de las mejoras tecnológicas, los filtros analógicos todavía poseen ciertas limitaciones en cuanto al cambio de las frecuencias de cortes y su cercanía a un comportamiento selectivo. Como alternativa se cuenta con los filtros digitales que ofrecen la comodidad de una fácil implementación y configuración de sus frecuencias de corte. Con respecto a los filtros digitales, este capítulo contiene una breve revisión de los sistemas básicos de filtrado digital; además de ejemplos de implementación y simulación, a través del asistente paquete computacional Matlab. Para poder evaluar el comportamiento de los filtros digitales en el contexto de la señal ECG, es de gran importancia conocer los tipos de distorsiones que se desean eliminar de esta señal.

Los filtros analógicos son implementados mediante la interconexión de dispositivos electrónicos y son orientados al tratamiento de señales en modo analógico. Sin embargo, en el caso de los filtros digitales es necesario que las 
señales analógicas sean convertidas en señales digitales. Los filtros digitales, a diferencia de los analógicos, son construidos a partir de algoritmos desarrollados en lenguajes de programación. Los filtros digitales se implementan, regularmente, a través de ecuaciones en diferencia (ver ecuación 1) compuestas por una serie de coeficientes $B_{k}$ que operan las muestras de la señal de entrada $x(n)$.

ECUACIÓN 1.

$$
y(n)=\sum_{k-0}^{L-1} B_{k} \cdot x(n-k)
$$

La ecuación 1 ejemplifica un sistema FIR para filtros digitales. Para la ejecución del filtro FIR es necesario tener la muestra de entrada actual y un número de muestras anteriores. Este tipo de sistemas puede adquirir la configuración de pasa bajos, pasa altos, pasa banda y rechaza banda. Los valores de los coeficientes $B_{k}$ se pueden calcular a través de la técnica de la ventana [30].

En Matlab ${ }^{\circledR}$ es posible implementar un algoritmo para el diseño de filtros pasa bajas. El primer paso es elegir el orden del filtro $L$, la frecuencia de muestreo Fs y las respectivas frecuencias de corte. A continuación se exponen las líneas de código con valores típicos de frecuencias de muestreo (Fs) y un valor de orden igual a 100.

En primera instancia se declaran las variables: frecuencia de muestreo (Fs), periodo de muestreo (Ts), orden del filtro (L), índice de muestras (n) y el valor de la frecuencia de corte $(\mathrm{Fc})$.

$$
\begin{aligned}
& F s=400 ; \\
& T s=1 / F s \\
& L=100 ; \\
& n=1: L ; \\
& F C=50 ;
\end{aligned}
$$

El segundo paso es generar la respuesta al impulso del filtro. Esta función contiene el grupo de coeficientes $B_{k}$ del filtro y proporcionan la característica pasa bajo del mismo. En este caso se generan los coeficientes que correspon- 
den a un filtro pasa bajas con valor de frecuencia de corte Fc. Este método se denomina técnica de la ventana.

$$
\begin{aligned}
& B=\left(\sin \left(2 * p i^{*} F c^{*} T s^{*}(n-(L / 2))\right)\right) . /(p i *(n-(L / 2))) ; \\
& k=L / 2 ; \\
& B(k)=1 ;
\end{aligned}
$$

Por medio de la transformada $z$ se realiza el análisis de la respuesta en frecuencia del filtro pasa bajas diseñado. La figura 13 contiene el comportamiento del filtro FIR pasa bajas. En Matlab, la transformada $z$ puede ser ejecutada a través de la función: freqz y requiere como entrada los coeficientes del filtro y el valor de la frecuencia de muestreo.
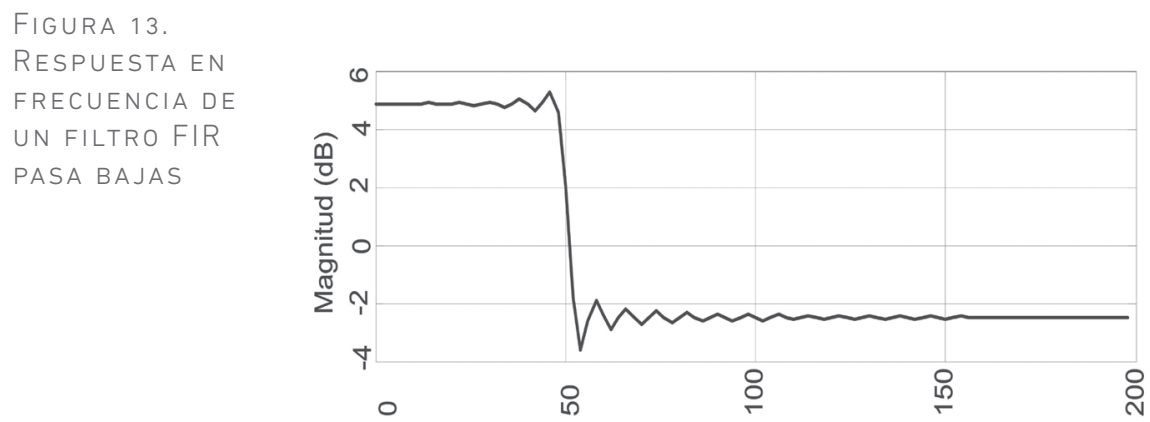

PROPIA.

Un filtro FIR pasa bajas tiene diversas aplicaciones en la señal ECG, principalmente la de aislar componentes espectrales de alta frecuencia. También permite generar un efecto de suavizado en la señal eliminando rizados.

Cuando es necesario eliminar componentes de muy baja frecuencia, como es el caso de componentes de continua, es requerido el uso de filtros digitales pasa altas. Para diseñar un filtro FIR pasa altas, por medio de la técnica de la ventana, se utilizan las siguientes líneas de código:

$$
\begin{aligned}
& F s=400 ; \\
& T s=1 / F s \\
& L=100 ; \\
& n=1: L
\end{aligned}
$$




$$
\begin{aligned}
& F C=0.7 \\
& B=\left(\sin \left(p i^{*}(n-(L / 2))\right)\right) . /\left(p i^{*}(n-(L / 2))-\left(\sin \left(2 * p i^{*} F C^{*} T s^{*}(n-(L / 2))\right)\right) .\right. \\
& /\left(p i^{*}(n-(L / 2))\right) \\
& k=L / 2 \\
& B(k)=1
\end{aligned}
$$

La figura 14 contiene la respuesta en frecuencia de un filtro pasa altas, generado a través de la función freqz.
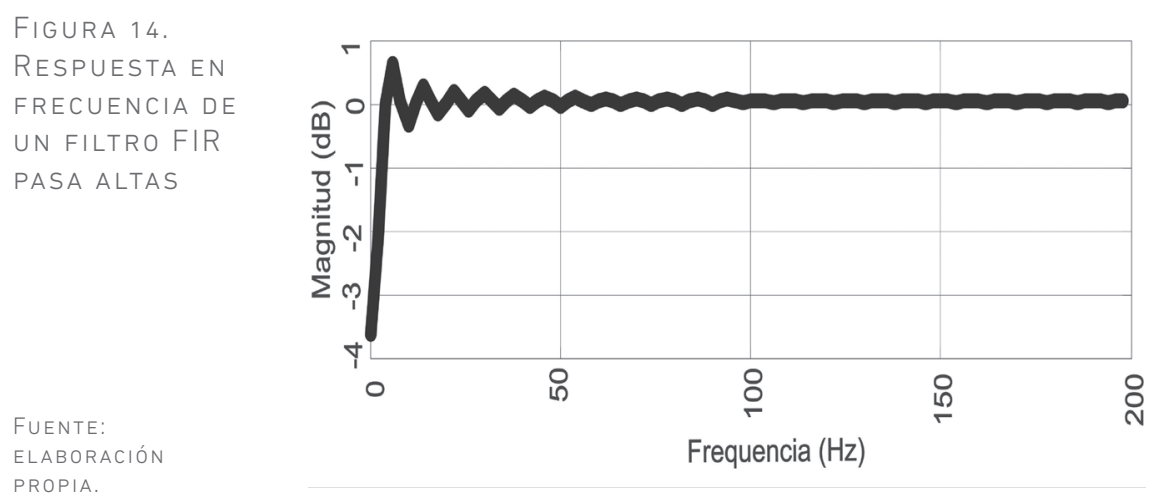

Frequencia $(\mathrm{Hz})$

Los filtros digitales FIR, pasa altas o pasa bajas, permiten operar las muestras adquiridas de un registro electrocardiográfico previamente digitalizado. En caso de requerir un cambio en los valores de frecuencia de corte, solo es necesario cambiar el valor de una variable y generar de nuevo el conjunto de coeficientes. No es requerido el cambio o adaptación de elementos electrónicos, como es el caso de los filtros analógicos.

\section{Desplazamiento de la línea de base}

El desplazamiento de línea de base es una distorsión que causa una oscilación en la componente de continua de la señal ECG. Esta oscilación puede causar distorsiones en las formas de ondas y su indebida detección. En la figura 15 se puede apreciar un ejemplo de señal ECG con desplazamiento de línea de base, tomada de la base de datos Physionet. 
FIGURA 15

SEÑAL ECG CON DESPLAZAMIENTO DE LÍNEA DE BASE

FUENTE: ELABORACIÓN PROPIA.

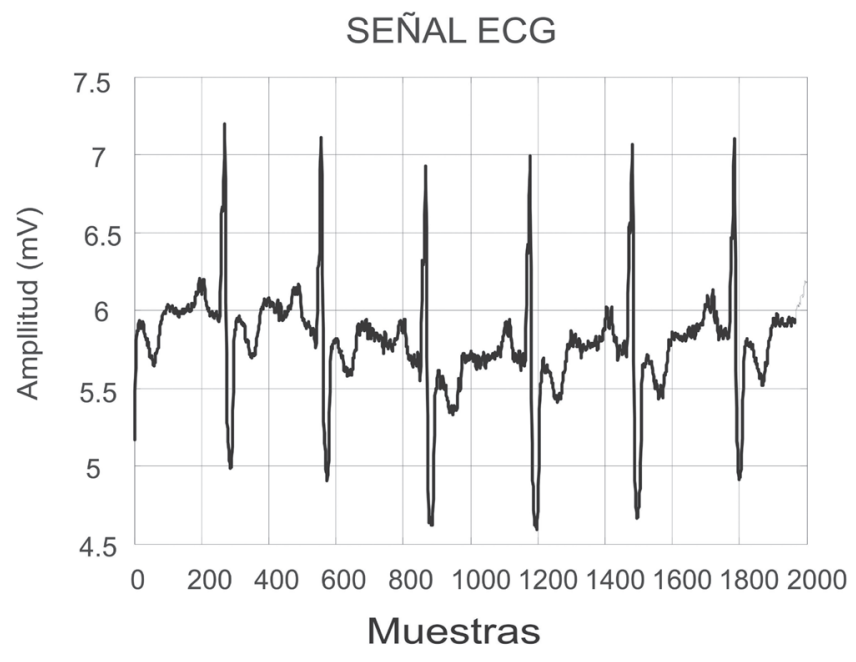

SEÑAL ECG

Una aplicación de filtros digitales FIR, en configuración de pasa altas, se ha utilizado en el tratamiento de la distorsión denominada: desplazamiento de la línea de base [31]. Esta distorsión se caracteriza por causar cambios en el valor de continua de la señal ECG. Tomando señales del repositorio Physionet [32] y con ayuda de Matlab ${ }^{\circledR}$, es posible simular el efecto que puede sufrir una señal ECG al ser distorsionada por un desplazamiento de línea de base.

Las señales obtenidas en el repositorio Physionet son tomadas de registros electrocardiográficos de sujetos reales durante el desarrollo de investigaciones. En el repositorio se puede contar con un gestor de registro digitales: chart - o - matic. Con el uso del gestor, los registros de señales ECG pueden ser exportados a archivos de diferentes formatos. En este caso es necesario el uso de archivos con extensión .mat, los cuales son de uso del asistente matemático Matlab.

A continuación se presenta un conjunto de líneas de código que permite ejecutar la simulación de una distorsión causada por el desplazamiento de línea de base. En esta simulación se toma el archivo ecg.mat, el cual contiene una señal ECG del repositorio Physionet. Posteriormente se genera una señal sinusoidal de muy baja frecuencia, que representa el ruido. De manera consecutiva se obtiene una versión obtenida de la suma de la señal ECG y el 
ruido simulado. Esta simulación se puede implementar usando las siguientes líneas de código:

$\%$ Cargar señal ECG almacenada en variable s en formato Mat.

loadecg

Fs $=125$;

Ts $=1 / f_{s}$;

$N=$ length(s);

$n=1: N$;

$t=(n-1) * T s ;$

$r 2=0.3^{*} \sin \left(2 * i^{*} 0.1 * n^{*}(T s)\right)$;

$x=s^{\prime}+r 2$;

La figura 16 contiene el resultado de la simulación que permite visualizar una señal ECG con desplazamiento de línea de base. En la parte superior de la figura 14 se puede observar el registro ECG original, tomado del repositorio Physionet y almacenado en el archivo ecg.mat. En la parte inferior (figura 16) se puede ver la nueva versión de la señal, la cual es el resultado de la adición del ruido simulado a la señal original.

FIGURA 16.

SEÑAL ECG CON

DESPLAZAMIENTO

DE LÍNEA DE

BASE SIMULADO
FUENTE:

ELABORACIÓN PROPIA.
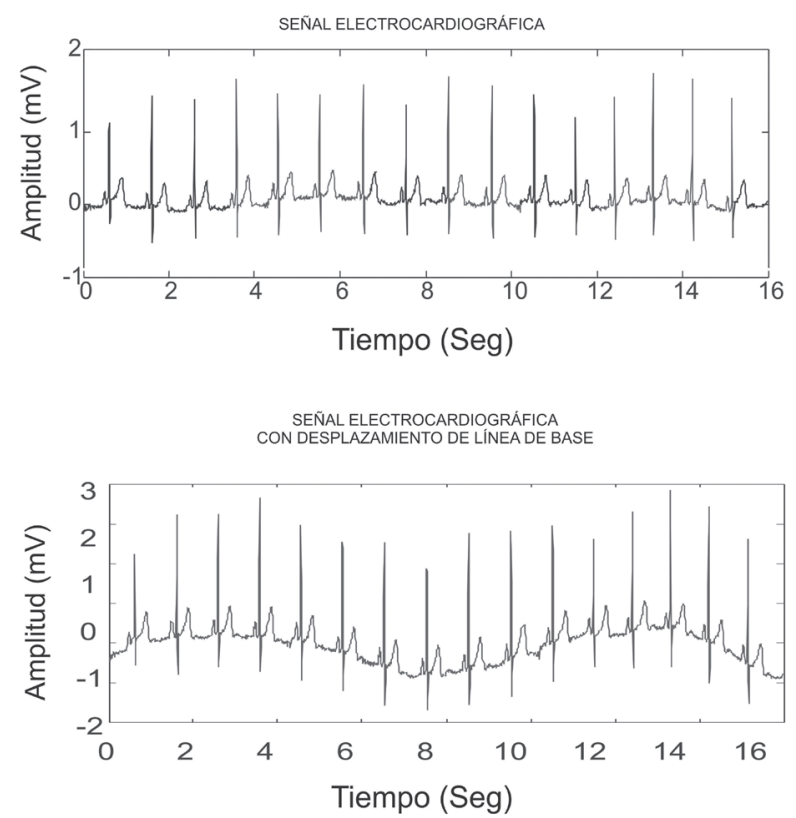
A través del comando filter se ejecuta el proceso de filtrado y se obtiene la señal de salida almacenada en la variable de salida (y). A continuación se presenta un ejemplo del uso del comando filter, el cual usa la variable $B$ que contiene los coeficientes del filtro FIR y la variable $x$ que representa la señal de entrada.

$$
y=\text { filter }(B, 1, x) \text {; }
$$

El resultado obtenido con el comando filter corresponde a una versión filtrada de la señal simulada. Esta nueva versión se puede observar en la figura 17. A pesar del proceso ejecutado, todavía se puede apreciar que persiste una componente de desplazamiento de línea de base.

FIGURA 17.

TRATAMIENTO DE DISTORSIÓN POR

DESPLAZAMIENTO

DE LIINEA DE

BASE A TRAVÉS

DE UN FILTRO

FIR PASA ALTAS

FUENTE:

ELABORACIÓN PROPIA.

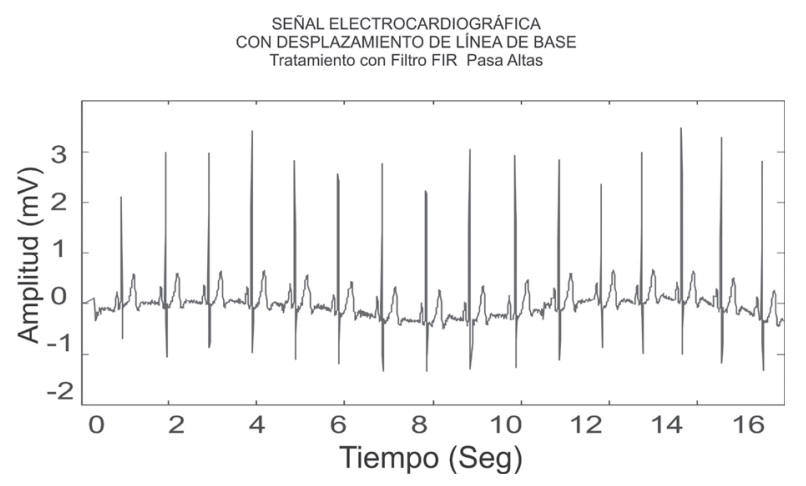

El resultado obtenido al tratar el desplazamiento de línea de base del ECG promedio de un filtro pasa altas es aceptable, ya que algunos componentes de bajas frecuencias se pueden atenuar. Se observa un retraso de grupo, que consiste en el desplazamiento de la señal en un número de muestras igual al orden del filtro pasa altos FIR. Este desplazamiento puede causar inconvenientes en el análisis de la señal ECG, ya que puede generar la perdida de distancia temporal entre cada una de sus ondas.

Como alternativa se puede diseñar un filtro digital con base en un sistema orientado a la cancelación de señales. Este tipo de sistema está basado en la resta entre la señal de entrada y el ruido estimado. La figura 18 ilustra el diagrama de bloques de un sistema de cancelación compuesto por una señal de 
entrada (en este caso es una señal ECG con desplazamiento de línea de base), un filtro pasa bajas y una etapa de sustracción.
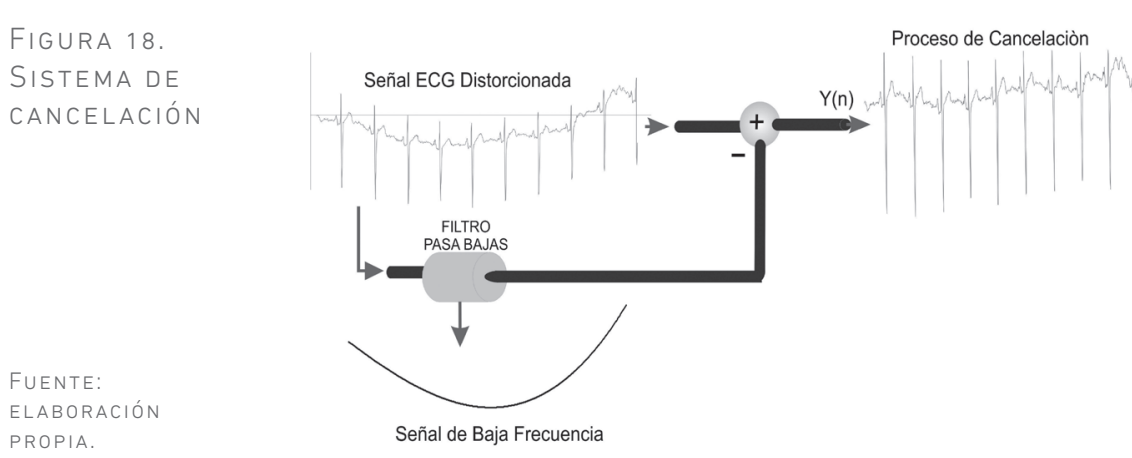

El papel que desempeña el filtro pasa bajas, consiste en extraer la componente de muy baja frecuencia que causa el desplazamiento de línea de base de la señal ECG. A través de líneas de código, se implementa un filtro pasa bajas con frecuencia de corte $F c=0.01 \mathrm{~Hz}$, que representa un valor típico de un ruido de muy baja frecuencia. Este código tiene los valores de $L$, que representa el orden del filtro y tiene la finalidad de extraer la componente de baja frecuencia que causa el desplazamiento de línea de base del ECG.

Para el desarrollo de este nuevo ensayo se ha optado por otra variedad de filtro pasa bajos. En este caso se denomina filtro promediador y consiste en un sistema discreto que genera una salida a partir del promedio con una cantidad $L$ de muestras anteriores.

En las siguientes líneas de código se declara un valor $L=16$, y posteriormente se genera un arreglo de datos B2. Este arreglo de datos posee una cantidad $L$ de unos, y cada uno es divido entre la variable $L$.

$$
\begin{aligned}
& L=16 ; \\
& B 2=(1 / L) * \text { ones }(1, L) ; \\
& y 2=\text { filter }(B 2,1, y 1) ;
\end{aligned}
$$

El resultado obtenido es una señal de muy baja frecuencia, que indica la tendencia del desplazamiento de línea de base. La figura 19 contiene el resultado obtenido. 
FIGURA 19.

TENDENCIA DEL

DESPLAZAMIENTO

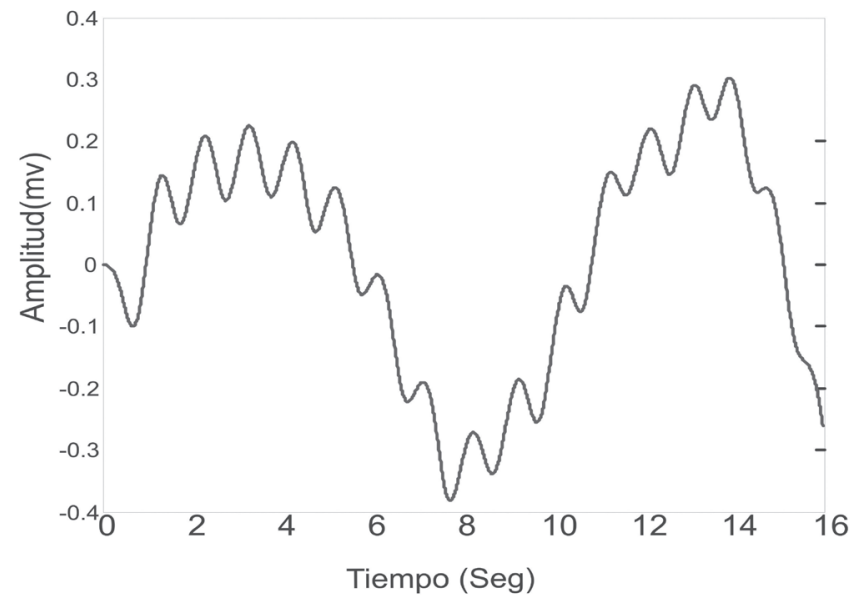

FUENTE:

ELABORACIÓN

PROPIA.

DE LÍNEA DE

BASE

El sistema de cancelación se ejecuta calculando la resta entre la señal de entrada y la señal de tendencia del desplazamiento de línea de base. La figura 20 ilustra el resultado de la diferencia entre ambas señales.

FIGURA 20.

TRATAMIENTO CON SISTEMA DE CANCELACIÓN

FuENTE:

ELABORACIÓN PROPIA.
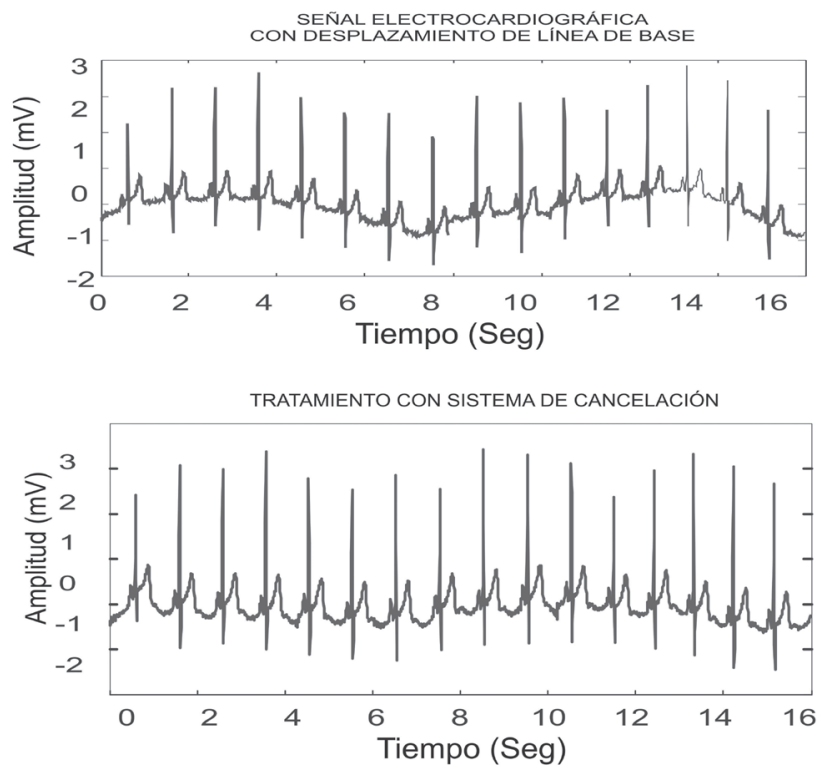
La ventaja del filtrado por cancelación es que permite la eliminación de un ruido sin causar distorsiones en componentes espectrales cercanos. Esta característica es importante en el tratamiento de la señal ECG, ya que en el uso de sistemas convencionales se pueden encontrar filtros digitales con características no ideales. Esto permite que sean eliminadas componentes espectrales cercanas a las propias del ruido, lo que conlleva la reducción de formas de ondas que transportan información importante en un proceso de diagnóstico [31].

\section{Ruido de línea de potencia eléctrica}

El ruido de línea de potencia eléctrica es el producto de los dispositivos eléctricos que se alimentan por medio de sistemas de corriente alterna con frecuencia de $60 \mathrm{~Hz}$ (sistema americano) o $50 \mathrm{~Hz}$ (sistema europeo). Este ruido es difícil de evitar, ya que los sistemas de alimentación de energía eléctrica son de vital importancia y están presentes en todas las infraestructuras físicas que contienen equipos orientados a la adquisición de señales electrofisiológicas. Este tipo de ruido se caracteriza por introducir rizados en la señal ECG, lo cual causa dificultad en la observación de sus formas de ondas.

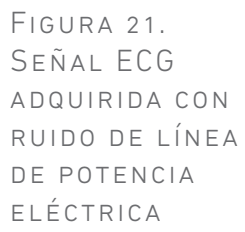

FUENTE:

ELABORACIÓN PROPIA.

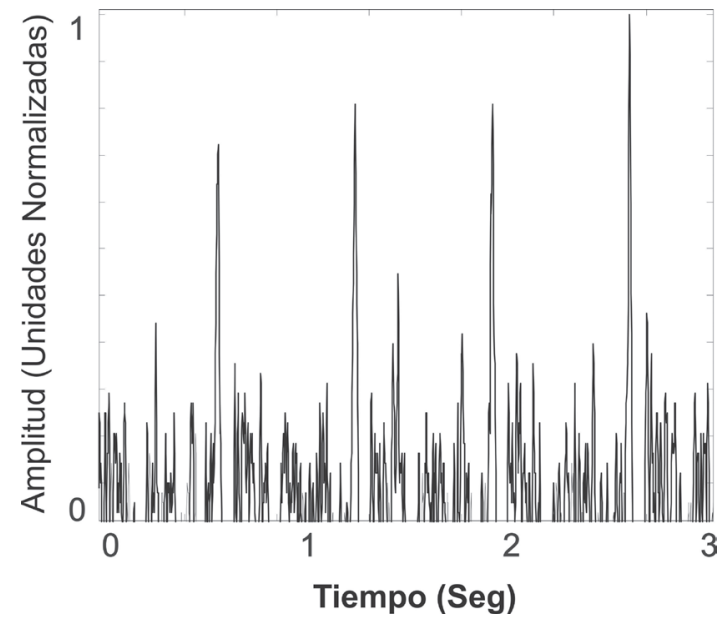


En primera instancia, el ruido de línea de potencia eléctrica era tratado a través de filtros analógicos rechaza banda o tipo muesca. Estos tipos de filtros tienen la función de especializarse en el rechazo de componentes espectrales con un valor de frecuencia definido. Con el auge de los filtros digitales, se ha preferido realizar el tratamiento de este ruido a través de medios computacionales.

Para poder ensayar estrategias de filtrado digital rechaza banda, es necesario poder tener un modelo del ruido que se requiere eliminar. En el caso del ruido de línea de potencia eléctrica, se puede modelar a través de un sencillo esquema basado en una señal sinusoidal con frecuencia única de 60 o $50 \mathrm{~Hz}$, según el sistema eléctrico. Tomando la señal ECG de la base de datos Physionet, almacenada en la variable $s$, se puede simular el efecto de distorsión causado por el ruido de línea de potencia. En este código se obtiene una nueva señal $x$, que contiene la suma de la señal ECG y una serie de tiempo con el modelo senosoidal del ruido de línea de potencia eléctrica.

El código sugerido es el siguiente:

$$
\begin{aligned}
& T s=0.008 ; \\
& F s=1 / T s ; \\
& N=\text { length }(s) ; \\
& n=1: N ; \\
& t=(n-1)^{*} T s ; \\
& r=0.1 * \sin \left(2^{*} \mathrm{pi}^{*} 60^{*} n^{*} T s\right) ; \\
& x=s+r ;
\end{aligned}
$$

La figura 19 contiene un ejemplo de distorsión causado por el ruido de línea de potencia eléctrica en una señal ECG. En la figura 22 se puede ver que la señal ECG posee un rizado, el cual es característico de la distorsión causada por el ruido de línea de potencia eléctrica.

Cabe mencionar que el ruido de línea de potencia eléctrica no es la única fuente de rizados en la señal ECG. Pueden existir otros ruidos con componentes espectrales de alta frecuencia que generan distorsiones similares, causadas por movimientos musculares o por otros sistemas fisiológicos. En estos escenarios es necesario utilizar análisis de la señal ECG en un dominio diferente al del tiempo. Un tipo de análisis es el basado en la Transformada 
FIGURA 22.

SIMULACIÓN DE UNA SEÑAL ECG CON RUIDO DE LÍNEA DE POTENCIA ELÉCTRICA

FUENTE: ELABORACIÓN PROPIA.

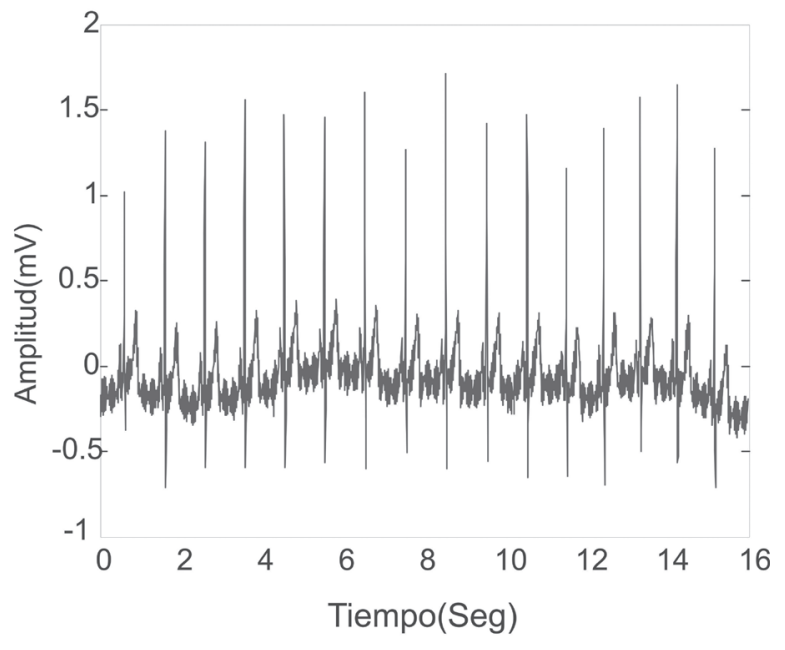

Rápida de Fourier (FFT, por sus siglas en inglés), el cual permite comparar la señal bajo estudio con modelos sinusoidales. La figura 20 contiene el espectro en magnitud de una señal ECG con ruido de línea de potencia eléctrica, calculado a través de la FFT.

Por medio de la FFT, se puede verificar cómo el espectro de una señal ECG evidencia la presencia de un ruido de línea de potencia. La figura 23 ilustra un pico en magnitud localizado en la frecuencia de $60 \mathrm{~Hz}$ en el espectro de la señal ECG.

FIGURA 23.

ESPECTRO EN MAGNITUD DE UNA SEÑAL ECG CON RUIDO DE LÍNEA DE POTENCIA ELÉCTRICA

FUENTE:

ELABORACIÓN PROPIA.

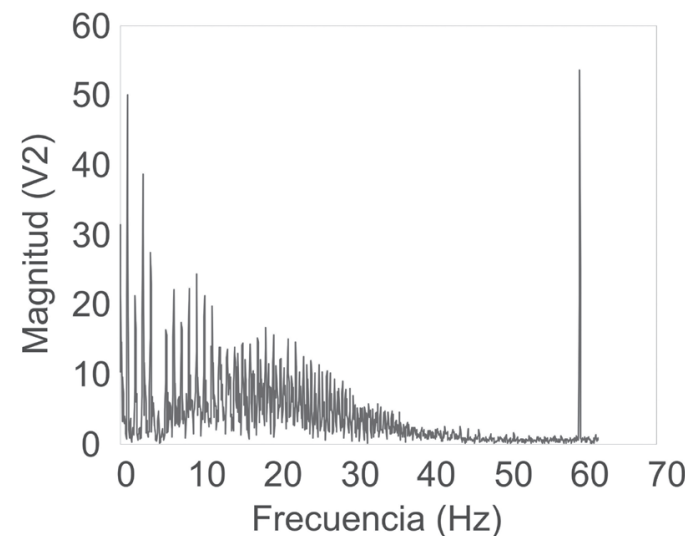


Una forma común de tratar el ruido de línea de potencia es a través de un filtro digital FIR tipo muesca (notch). Por medio de la técnica de diseño de filtros FIR, basada en la colocación de ceros, se genera una variable $B 1$ que contiene el valor de la frecuencia que se requiere atenuar [33].

Se pueden utilizar las siguientes líneas de código:

$$
\begin{aligned}
& B 0=1 \text {; } \\
& B 2=1 \text {; } \\
& B 1=-2^{*} \cos \left(2^{*} \mathrm{pi}^{*}(60 /(\mathrm{Fs}))\right) \text {; } \\
& B=\left[\begin{array}{llll}
B 0 & B 1 & B 2
\end{array}\right] \text {; }
\end{aligned}
$$

La figura 24 contiene el análisis en el dominio de la frecuencia del filtro FIR tipo muesca para eliminar una componente espectral de $60 \mathrm{~Hz}$.
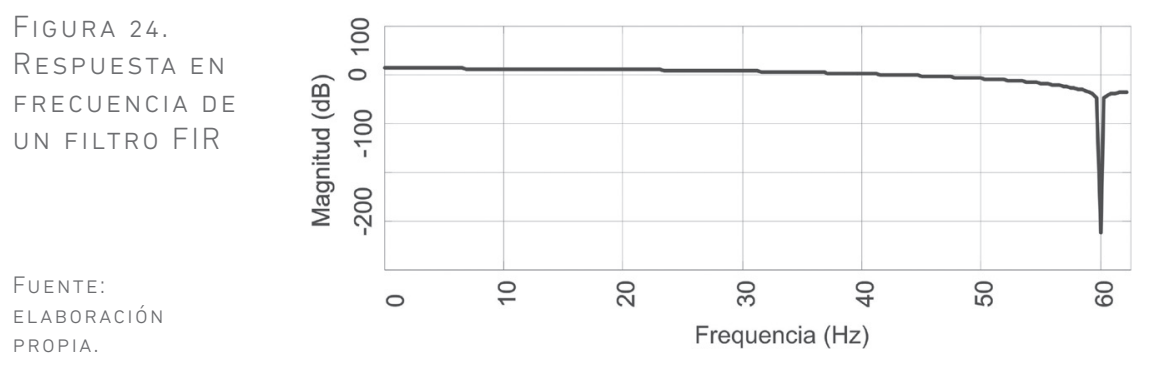

En el análisis del dominio de la frecuencia del filtro digital tipo Notch (figura 21), se puede observar una atenuación de más de 200 decibeles en la frecuencia de $60 \mathrm{~Hz}$. De todas formas, también se puede observar que el filtro diseñado no es ideal, lo que se puede evidenciar en las atenuaciones que puede generar en componentes espectrales cercanas a la frecuencia del ruido.

La figura 25 ilustra el resultado obtenido al utilizar el filtro FIR muesca sobre la señal ECG. Se puede apreciar, en el espectro en magnitud, la ausencia de la componente de $60 \mathrm{~Hz}$. El resultado contenido en la figura 22 también permite evidenciar que el resultado no viene de un filtro digital ideal y se puede notar en la presencia de algunos rizados a lo largo de la señal ECG obtenida. 
FIGURA 25.

TRATAMIENTO

DE UN ECG

EJECUTADO CON

UN FILTRO FIR

MUESCA

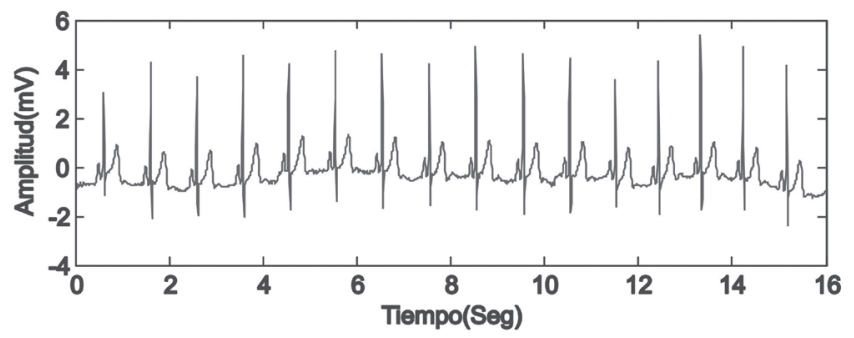

FUENTE:

ELABORACIÓN

PROPIA.

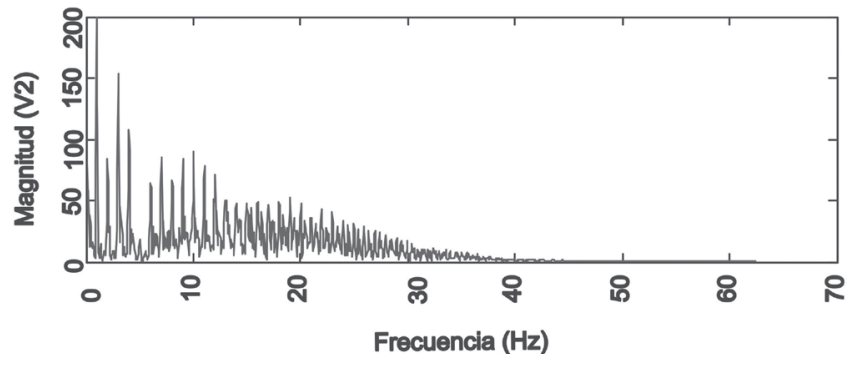

Los sistemas de acondicionamiento de señales cumplen la tarea de amplificar y establecer el ancho de banda de la señal ECG. Esto garantiza el rechazo de posibles ruidos con componentes espectrales de frecuencia externas al ancho de banda propio de la señal ECG.

Pero, teóricamente, se ha estudiado que muchos ruidos que causan distorsiones en la señal ECG poseen componentes espectrales con valores de frecuencia contenidos en el ancho de banda de la señal bajo estudio. Esto no permite una debida atenuación por parte del sistema de acondicionamiento de señales.

En este capítulo se ha estudiado los sistemas convencionales de filtrados digitales, los cuales son implementados a través de algoritmos basados en ecuaciones en diferencia (ver ecuación 1). Estos algoritmos operan sobre las muestras obtenidas del proceso de conversión análogo a digital de la señal ECG.

Los sistemas basados en filtros digitales FIR cumplen una labor importante que consiste en atenuar las distorsiones causadas por señales no deseadas, las cuales no son debidamente atenuadas por el sistema de acondicionamiento de señales. Los filtros FIR son de fácil implementación y requieren el uso 
de la muestra actual de entrada y las muestras anteriores. La cantidad de muestras anteriores requeridas dependen del orden del filtro FIR escogido.

Como se pudo ver en este capítulo, los filtros digitales son diseñados bajo una estrategia matemático que está en función de la frecuencia de muestreo y los valores de frecuencia de corte. Esta estrategia matemática da como resultado una serie de coeficientes que hacen parte de la ecuación en diferencias. Este esquema ofrece estabilidad y una respuesta en tiempo y frecuencia estática. Una gran debilidad de los filtros digitales es causada por su comportamiento estático, el cual no tiene la capacidad de adaptarse ante los cambios en tiempo y frecuencia que pueda tener una interferencia.

La transformada $z$, utilizada a través del comando freqz, es de gran importancia en el proceso de diseño de filtros digitales FIR. Este análisis permite observar el comportamiento en el dominio de la frecuencia del filtro, permitiendo determinar las atenuaciones en las bandas de paso y de rechazo.

Como se puede ver en las simulaciones (figuras 25 y 21), los filtros digitales convencionales no siempre logran atenuar las distorsiones. Por lo cual es necesario contemplar esquemas avanzados de filtrado digital que tengan la capacidad de adaptar su comportamiento a los cambios dinámicos de cada interferencia.

Un ejemplo de sistemas alternativos de filtrados es mostrado en este capítulo y está basado en la cancelación de señales. Este tipo de sistemas requiere de la generación de una señal de referencia que estima el comportamiento del ruido que causa la distorsión de la señal ECG.

El siguiente capítulo presenta otras alternativas de sistemas de filtrados con capacidad de adaptación. Específicamente se abordará el esquema de los filtros adaptativos. 
